\title{
Optimal 3GPP Fairness Parameters in 5G NR Unlicensed (NR- U) and WiFi Coexistence
}

This paper was downloaded from TechRxiv (https://www.techrxiv.org).

\section{LICENSE}

CC BY 4.0

SUBMISSION DATE / POSTED DATE

$16-02-2022$ / 22-02-2022

\section{CITATION}

Kakkad, Yashraj; Patel, Dhaval; Kavaiya, Sagar; Sun, Sumei; Lopez-Benitez, Miguel (2022): Optimal 3GPP Fairness Parameters in 5G NR Unlicensed (NR-U) and WiFi Coexistence. TechRxiv. Preprint. https://doi.org/10.36227/techrxiv.19180787.v1

$\mathrm{DOI}$ 


\title{
Optimal 3GPP Fairness Parameters in 5G NR Unlicensed (NR-U) and WiFi Coexistence
}

\author{
Yashraj Kakkad, Student Member, IEEE, Dhaval K Patel, Member, IEEE, Sagar Kavaiya, \\ Sumei Sun, Fellow, IEEE, and Miguel López-Benítez, Senior Member, IEEE
}

\begin{abstract}
The fifth-generation networks, designed to provide a better quality of service and spectrum utilization, are rapidly being deployed across the world. 3GPP has proposed a fairness criterion, referred to as "3GPP fairness", for the coexistence of 5G New Radio in unlicensed spectrum (NR-U) and WiFi in Release 16. In this correspondence, we derive an analytical expression to determine if the 3GPP fairness is achieved in a given network configuration. We model achieving the 3GPP fairness as an optimization problem and show how to use Sequential Quadratic Programming to find the optimal 5G NR-U parameters from a pre-decided range. We test the optimizer in various conditions and inspect the effect of various parameters. Our results reveal that the optimizer is able to predict the best possible parameters for 3GPP fairness and therefore the proposed method proves useful for tuning 5G NR-U parameters during their coexistence with WiFi.
\end{abstract}

Index Terms-5G NR-U, WiFi, coexistence, 3GPP fairness, cellular, spectrum sharing, resource management

\section{INTRODUCTION}

$3^{\mathrm{n}}$ RD Generation Partnership Project (3GPP) has standardized 5G NR-Unlicensed (NR-U) radio access technology in the Release 16 [1], as the successor of LTE-Licensed Assisted Access (LAA) introduced in Release 13 [2]. 5G NR-U will coexist with other technologies in the unlicensed spectrum, notably WiFi. One of the key concerns to be addressed is setting appropriate ground rules for channel acquisition to ensure quality of service (QoS) for both $5 \mathrm{G}$ NR-U and WiFi users. 3GPP has proposed their definition of fairness in Release 16 [1] for 5G NR-U similar to that of LTE-LAA [2], which restricts the WiFi performance to not be reduced by intrusion of NR-U (or LTE-LAA). The LTE-LAA definition has received some criticism in literature because of its one-sided nature, such as in [3]-[4]. Authors in [5] have demonstrated how 3GPP fairness can be achieved in LTE-U and WiFi coexistence by appropriately regulating the duty cycle. Study in [6] has shown that channel access mechanisms can be designed for enabling coexistence where 3GPP fairness is achieved. The work presented in [7] proposed a QoS aware mechanism which has a fairness constraint. It has been shown in [8] that, in at least the common scenarios, 3GPP fairness is satisfied.

Given that 3GPP fairness may not be achieved in every network scenario [4], it would be useful to determine the optimal 5G NR-U parameters such that fairness criterion is met

Manuscript received on July 02, 2021. Yashraj Kakkad, Dhaval K Patel and Sagar Kavaiya are with School of Engineering and Applied Science, Ahmedabad University, India. (Email:kakkad.m@ahduni.edu.in, dhaval.patel@ahduni.edu.in, sagar.k@ahduni.edu.in). Sumei Sun is with Institute of Infocomm Research, Singapore. (Email:sunsm@i2r.a-star.edu.sg) Miguel López-Benítez is with the Department of Electrical Engineering and Electronics, University of Liverpool, Liverpool, L69 3GJ, United Kingdom, and also with the ARIES Research Centre, Antonio de Nebrija University, 28040 Madrid, Spain (Email: m.lopez-benitez@liverpool.ac.uk). as closely as possible. To the best of our knowledge, limited work has been published for coexistence of $5 \mathrm{G}$ NR-U with $\mathrm{WiFi}$, and there is no work in literature to tune an arbitrary number of $5 \mathrm{G}$ NR-U parameters to achieve 3GPP fairness. Our key contributions in this paper are as follows:

1) We express the problem of achieving 3GPP fairness as an optimization problem with a non-linear objective function and constraints. We show how the Sequential Quadratic Programming algorithm [9] can be used to solve the problem by tuning $5 \mathrm{G}$ NR-U parameters and obtain the "fairest" situation where WiFi and 5G NR-U throughput are the closest possible.

2) We inspect the effect of various $5 G$ NR-U parameters on the 3GPP criterion and demonstrate the validity of the proposed approach.

\section{SYSTEM MODEL}

We consider the system model mentioned in [3], which is an extension of [10] for LTE-LAA and WiFi coexistence. Fig. 1 depicts an illustration. The main difference is the variable $5 \mathrm{G}$ NR-U slot duration and sub-carrier spacing because of $5 \mathrm{G}$ flexible numerology. For the sake of brevity the entire system model is not repeated here. We consider $n^{(W)}$ WiFi nodes, in which $n^{(W)}-1$ stations are in association with an access point (AP), and $n^{(L)} 5 \mathrm{G}$ NR-U nodes, in which $n^{(L)}-1$ user equipment are associated with a gNodeB. The nodes of the same type $\Psi$, where $\Psi \in\{W, L\}$ have identical parameters as summarized in Table I.

We assume that nodes follow an independent timehomogeneous backoff process with an identical steady-state probability of successful transmission of head-of-line (HOL) packets as explained in [10]. The assumption is valid as long as the difference between the number of sensing slots of the nodes is reasonably small. The behaviour of the HOL packets is modeled as a discrete-time Markov renewal process with three categories of states - transmission $(T)$, backoff $\left(R_{i}\right)$ and collision $\left(F_{i}\right)$ where $i=0, \cdots, P^{(\Psi)}+Q^{(\Psi)}-1$. The state diagram is as shown in Fig. 2. Though the definition of a slot in $5 \mathrm{G}$ NR-U differs from LTE-LAA, we can consider the 5G NR-U slot duration $\left(D^{(L)}\right)$ instead of the LTE-LAA counterpart when computing the holding times of gNBs [11].

A WiFi node, upon accessing the channel transmits a $P L^{(W)}$ bits payload at $R^{(W)}$ Mbps transmission rate. On the other hand, a 5G NR-U node will transmit for a time duration of maximum channel occupation time (MCOT). We consider some standard assumptions found in literature [3] [4] [10]. The nodes are saturated and the channel is noiseless. i.e., there is no random error. The hidden node problem is assumed to not occur. Also, each node is assumed to have an infinite 


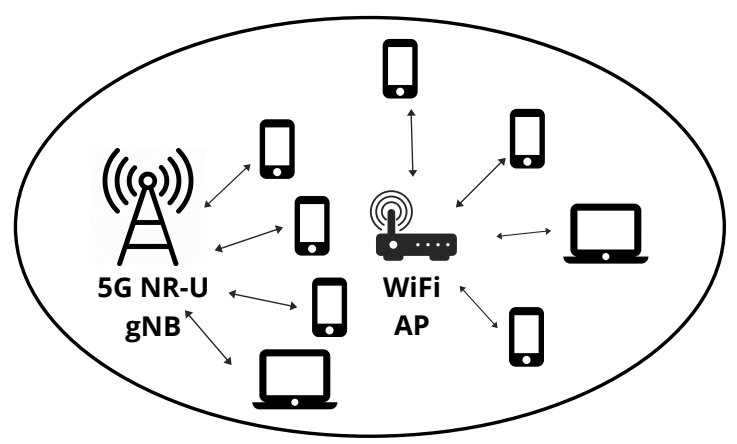

Fig. 1. Illustration of our coexistence system model.

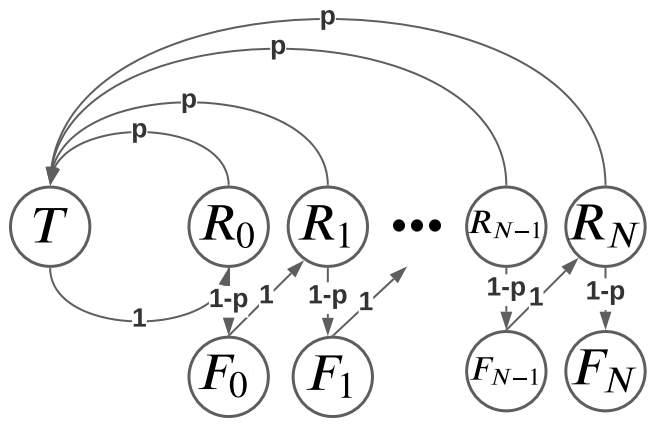

Fig. 2. State transition Markov chain of an individual HOL packet, $\Psi \in\{W, L\}, N=P^{(\Psi)}+Q^{(\Psi)}-1$

TABLE I

SySTEM PARAMETERS OF WIFI AND 5G NR-U

\begin{tabular}{c||c}
\hline Parameter & Notation \\
\hline \hline Initial backoff window size & $C^{(\Psi)}$ \\
Maximum backoff stage & $P^{(\Psi)}$ \\
Retry limit & $Q^{(\Psi)}$ \\
Number of sensing slots & $A^{(\Psi)}$ \\
Time duration of a successful transmission & $\tau_{T}^{(\Psi)}$ \\
Time duration of a failed transmission & $\tau_{F}^{(\Psi)}$ \\
\hline
\end{tabular}

buffer. Let $\alpha^{(\Psi)}$ be the steady state probability of the channel being idle. For the sake of brevity, we omit some derivations mentioned in [3]. Consider the derived expression of the probability of a HOL packet of type $\Psi$ transmitting:

$$
\begin{gathered}
\tilde{\pi}_{T}^{(\Psi)}=\frac{\tau_{T}^{(\Psi)}}{\tau_{T}^{(\Psi)}+\tau_{F}^{(\Psi)} \cdot\left(\frac{1-p}{p}\right)+\left(\frac{\Sigma_{i=0}^{P^{(\Psi)}+Q^{(\Psi)}-1}(1-p)^{i} \cdot \frac{1+C_{i}^{(\Psi)}}{2}}{\alpha^{(\Psi)} \cdot\left(1-(1-p)^{P^{(\Psi)}+Q^{(\Psi)}}\right)}\right)}, \\
C_{i}^{(\Psi)}=C^{(\Psi)} \cdot 2^{\min \left\{i, P^{(\Psi)}\right\}}
\end{gathered}
$$

$C_{i}^{(\Psi)}$ is the backoff window size at the $i$ th iteration, $0 \leq i \leq$ $P^{(\Psi)}+Q^{(\Psi)}-1$, Let $A_{\min }=\min \left(A^{(W)}, A^{(L)}\right) \cdot p$ is the steady state probability of successful transmission of a packet and the solution of the following approximation:

$$
p \approx \exp \left\{-\Sigma_{\Psi \in\{W, L\}} \frac{2 n^{(\Psi)} \cdot \frac{1-(1-p)^{P^{(\Psi)}+Q^{(\Psi)}}}{p^{A_{\min } A^{(\Psi)+1}}}}{\sum_{i=0}^{P^{(\Psi)}+Q^{(\Psi)}}(1-p)^{i} \cdot C_{i}^{(\Psi)}}\right\}
$$

It can be shown that a solution to the above equation exists in the range $0 \leq p \leq 1$. Expression (3) can be solved using suitable root finding techniques such as classic Brent's method [12].

Let $\alpha$ be the steady state probability of the channel being idle when $A^{(W)}=A^{(L)}$. It is related to its general counterpart by the following expression:

$$
\alpha^{(\Psi)}=\alpha \cdot p^{A^{(\Psi)}-A_{\min }}
$$

The below expression can be derived [3]

$$
\begin{array}{r}
\alpha=1 /\left(1+\tau_{F, \min } \cdot(1-p)+\Delta_{\tau_{F}} \cdot\left(1-\exp \left(\frac{\ln p \cdot X^{(\Psi)}}{\sum_{\Psi \in\{W, L\}} X^{(\Psi)}}\right)\right)\right. \\
\left.-\frac{\sum_{\Psi \in\{W, L\}} X^{(\Psi)}\left(\tau_{T}^{(\Psi)}-\tau_{F}^{(\Psi)}\right)}{\Sigma_{\Psi \in\{W, L\}} X^{(\Psi)}} \cdot p \ln p\right)
\end{array}
$$

Here $\tau_{F, \min }=\min _{(\Psi \in\{W, L\})} \tau_{F}^{(\Psi)}, \psi=\arg \max _{(\Psi \in\{W, L\})} \tau_{F}^{(\Psi)}$, $\Delta_{\tau_{F}}=\left|\tau_{F}^{(L)}-\tau_{F}^{(W)}\right|$ and

$$
X^{(\Psi)}=\frac{\frac{n^{(\Psi)} p^{A^{(\Psi)}}}{C^{(\Psi)}} \cdot\left(1-(1-p)^{P^{(\Psi)}+Q^{(\Psi)}}\right)}{\left(\frac{1}{2 p-1}+\left(\frac{1}{p}-\frac{1}{2 p-1}-\frac{(1-p) Q^{(\Psi)}}{p}\right)(2-2 p)^{P^{(\Psi)}}\right)}
$$

\section{3GPP FAIRNESS IN 5G NR-U AND WIFI COEXISTENCE}

\section{A. Derivation of the condition for achieving 3GPP fairness}

3GPP's definition of fairness [1] states that the NR-U design should target fair coexistence with existing WiFi networks to not impact WiFi services more than an additional WiFi network on the same carrier with respect to throughput and latency. A similar definition was proposed for LTE-LAA [2], for which the authors in [3] and [4] argue, the definition is in favour of WiFi, treating LTE-LAA as an 'intruder' or outsider in the spectrum. We consider the definition for the 5G NR-U case and slightly modify the 'per user throughput' considered in [4]. A similar approach is followed in [5] for coexistence of LTE-U and WiFi. Consider the following scenarios, having the same total number of $n$ nodes:

- Network A, consisting of a WiFi AP and $n-1$ stations.

- Network B, consisting of $x$ WiFi nodes and $n-x 5 \mathrm{G}$ NR-U nodes $(1 \leq x<n)$.

Considering the definition, 3GPP fairness is achieved if the average per-node throughput of Network B is not lower than the average per-node throughput in Network A [4], [5]. There is no direct condition imposed on 5G NR-U network.

We can now derive an expression to validate if $3 \mathrm{GPP}$ fairness is achieved given the network configuration. Node airtime being a measure of throughput can be used in the definition. Let the node fraction-of-time in the network be $\lambda_{\text {out }}^{(\Psi)}(\Psi \in\{W, L\})$. In a saturated condition, $\lambda_{\text {out }}^{(\Psi)}$ will be equal to its steady state probability of transmission (i.e., service rate):

$$
\lambda_{\text {out }}^{(\Psi)}=\tilde{\pi}_{T}^{(\Psi)}
$$

Let the total time of observation be $T$ units. We use the suffixes $A$ and $B$ to differentiate between the parameters of the two network models discussed above. For 3GPP fairness, 


$$
\begin{gathered}
\lambda_{\text {out }_{A}}^{(W)} \cdot T \leq \lambda_{\text {out }_{B}}^{(W)} \cdot T \\
\therefore \lambda_{\text {out }_{A}}^{(W)} \leq \lambda_{\text {out }_{B}}^{(W)}
\end{gathered}
$$

Assuming a saturated network:

$$
\tilde{\pi}_{T_{A}}^{(W)} \leq \tilde{\pi}_{T_{B}}^{(W)}
$$

Using (1), we obtain:

$$
\begin{aligned}
& \tau_{F}^{(W)} \cdot \frac{1-p_{A}}{p_{A}}+\left(\frac{\sum_{i=0}^{P^{(W)}+Q^{(W)}-1}\left(1-p_{A}\right)^{i} \cdot \frac{1+C_{i}^{(W)}}{2}}{\alpha^{(W)} \cdot\left(1-\left(1-p_{A}\right)^{P^{(W)}+Q^{(W)}}\right)}\right) \\
& \geq \tau_{F}^{(W)} \cdot \frac{1-p_{B}}{p_{B}}+\left(\frac{\sum_{i=0}^{P^{(W)}+Q^{(W)}-1}\left(1-p_{B}\right)^{i} \cdot \frac{1+C_{i}^{(W)}}{2}}{\beta \cdot\left(1-\left(1-p_{B}\right)^{P^{(W)}+Q^{(W)}}\right)}\right)
\end{aligned}
$$

The steady-state probability of sensing the channel idle in WiFi only scenario, $\beta$ can be obtained as [10]:

$$
\beta=\frac{1}{1+\tau_{F}-\tau_{F} p-\left(\tau_{T}-\tau_{F}\right) p \ln p}
$$

3GPP fairness can be achieved if the inequality in (10) is satisfied.

\section{B. Achieving 3GPP fairness by tuning $5 G N R-U$ parameters}

In this subsection, we are interested in determining if 3GPP fairness can be achieved by tuning a set of 5G NR$\mathrm{U}$ parameters (backoff window size, number of sensing slots, number of nodes, etc.) in a desired range. We now show that achieving 3GPP fairness can be modeled as an optimization problem. From the definition, consider the below function,

$$
F\left(P, p_{B}\right)=\underset{P}{\operatorname{minimize}}\left|\lambda_{\text {out }_{A}}^{(W)}-\lambda_{\text {out }_{B}}^{(W)}\right|
$$

where $P=\left[P_{1}, P_{2} \cdots P_{l}\right]$ denotes a vector of $5 \mathrm{G}$ NR-U parameters considered for tuning, which can be one or more from Table I, along with the $5 \mathrm{G}$ NR-U slot duration.

$p_{B}$, which is constrained by (3), must at the same time satisfy the inequality $0 \leq p_{B} \leq 1$. For practical purposes, it is reasonable to argue that $3 \mathrm{GPP}$ fairness is achieved when the optimized value is less than a predefined small threshold. Its value must be decided as per the network parameters and the tolerance.

We define the following functions for modeling our problem. Consider,

$$
G\left(P, p_{B}\right)=p_{B}-\exp \left\{-\sum_{\Psi \in\{W, L\}} \frac{2 n^{(\Psi)} \cdot \frac{1-\left(1-p_{B}\right)^{P^{(\Psi)}+Q^{(\Psi)}}}{p_{B}^{A_{m i n}-A^{(\Psi)}+1}}}{\sum_{i=0}^{P^{(\Psi)}+Q^{(\Psi)}}\left(1-p_{B}\right)^{i} \cdot C_{i}^{(\Psi)}}\right\}
$$

$$
\begin{aligned}
& q_{0}\left(p_{B}\right)=p_{B} \\
& q_{1}\left(p_{B}\right)=1-p_{B}
\end{aligned}
$$

Our problem can, therefore, be expressed as:

$$
\begin{aligned}
\operatorname{minimize} & F\left(P, p_{B}\right) \\
\text { subject to } & q_{0}\left(p_{B}\right) \geq 0 \\
& q_{1}\left(p_{B}\right) \geq 0 \\
& G\left(P, p_{B}\right)=0 \\
& g_{i}(P) \geq 0 \forall i \in[1, t]
\end{aligned}
$$

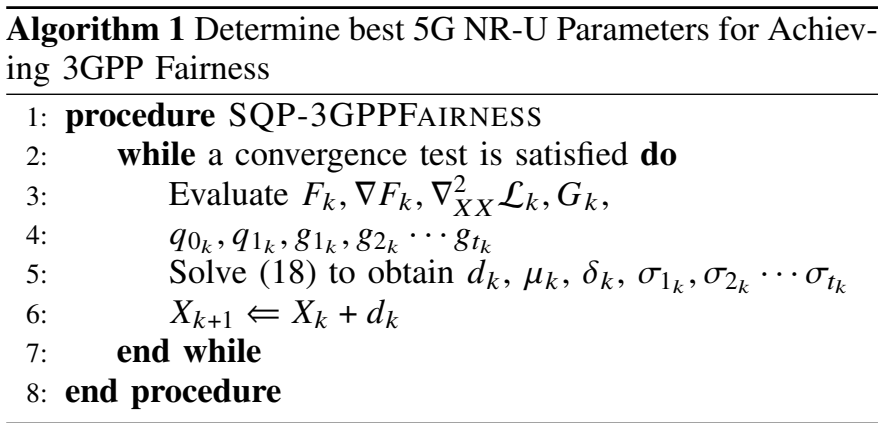

Here, $g_{i}(P)$ denotes the set of functions (distinguished by $i$ ) which define the bounds on the values of the parameters considered, analogous to (14) and (15). The Lagrangian function for this problem can be expressed as

$$
\begin{aligned}
\mathcal{L}\left(P, p_{B}, \lambda, \mu, \delta, \sigma_{0} \cdots \sigma_{k}\right) & =F\left(P, p_{B}\right)-\lambda^{T} q_{0}\left(p_{B}\right) \\
& -\mu^{T} q_{1}\left(p_{B}\right)-\delta^{T} G(P) \\
& -\Sigma_{i=0}^{t} \sigma_{i}^{T} g_{i}(P)
\end{aligned}
$$

Problems of the above form can be solved using Sequential Quadratic Programming (SQP) method [9], a class of algorithms for solving nonlinear optimization problems. Let $X_{k}$ denote $F$ 's parameters at the $k$ th iteration. Similarly, let the subscript $k$ in a function denote its value at the corresponding iteration. SQP determines the direction of search $d_{k}$ as the solution of the subproblem [9]:

$$
\begin{array}{cl}
\underset{d}{\operatorname{minimize}} & F_{k}+\nabla F_{k}^{T} d+\frac{1}{2} d^{T} \nabla_{X X}^{2} \mathcal{L}_{k} d \\
\text { subject to } & \nabla G\left(P_{k}, p_{B}\right)^{T} d+G\left(P_{k}, p_{B}\right)=0 \\
& \nabla q_{0}\left(p_{B_{k}}\right)^{T} d+q_{0}\left(p_{B_{k}}\right) \geq 0 \\
& \nabla q_{1}\left(p_{B_{k}}\right)^{T} d+q_{1}\left(p_{B_{k}}\right) \geq 0 \\
& \nabla g_{i}\left(p_{B_{k}}\right)^{T} d+g_{i}\left(p_{B_{k}}\right) \geq 0 \forall i \in[1, t]
\end{array}
$$

The above expression is a quadratic programming problem and can be solved using standard techniques [9]. The SQP algorithm for our context is described as Algorithm 1. In a static network configuration, the parameters can be computed in advance. In the contrary case, the gNB should obtain the WiFi parameters from a central authority or through crosstechnology communication. An approach for the latter for LTE-U and WiFi coexistence has been proposed in [13], discussion of which is beyond the scope of this work. Existing implementations can be referred to for an efficient incorporation in a practical system, for example a software package for SQP described in [14].

\section{NumERICAL RESUlTS}

We validate numerically our analysis, and the set of parameters generated by Algorithm 1. The results involving one varying parameter have been verified using Monte Carlo simulations in MATLAB, which are represented as dots in the subsequent figures. We execute each experiment for $10^{8}$ time slots, averaging each result 11 times for accurate results. The parameters are summarized in Table II. Packet arrival in each of the nodes is simulated as a Bernoulli process in each node with a fixed rate $\lambda^{(\Psi)}$ per type of node per iteration. $\lambda^{(\Psi)}$ is 


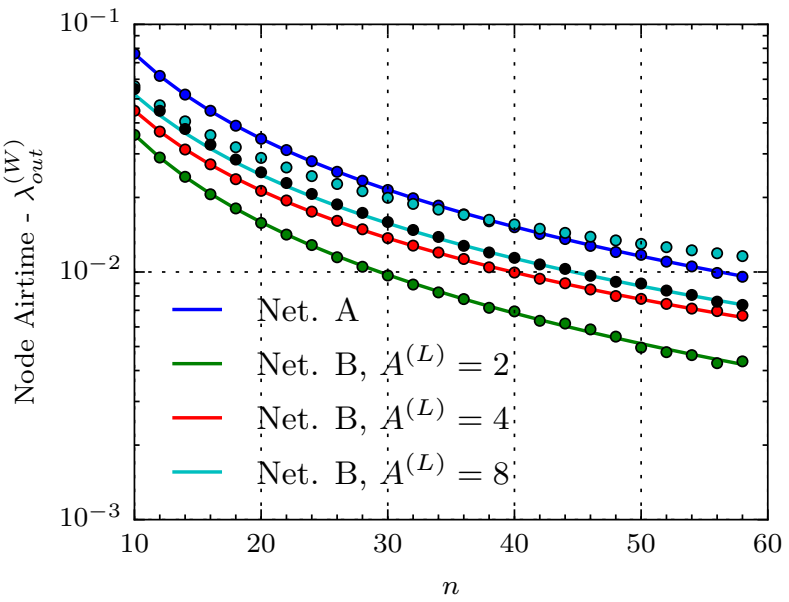

Fig. 3. Comparison of node airtime in networks $\mathrm{A}$ and $\mathrm{B}$ on varying $n$ with different values of $A^{(L)}$.

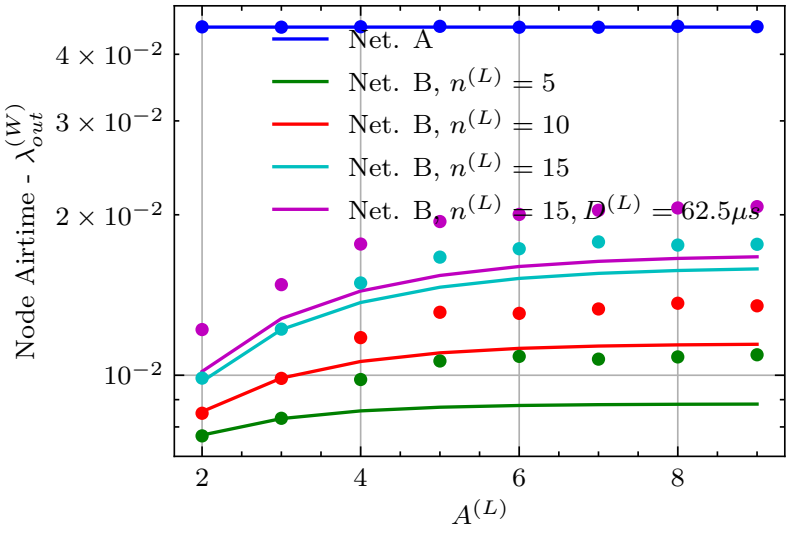

Fig. 5. Comparison of node airtime in networks $\mathrm{A}$ and $\mathrm{B}$ on varying $A^{(\cdot)}$.

TABLE II

SimUlation PARAMETERS

\begin{tabular}{c||c}
\hline PHY Header & $20 \mu s$ \\
ACK & 112 bits+PHYH \\
DIFS & $34 \mu s$ \\
SIFS & $16 \mu s$ \\
Slot Time - $\sigma$ & $9 \mu s$ \\
Basic Rate - $R_{B}$ & $6 \mathrm{Mbps}$ \\
\hline Packet Payload Length - PL $L^{(W)}$ & $2^{16} \mathrm{bits}$ \\
Data Rate - $\boldsymbol{R}^{(W)}$ & $24 \mathrm{Mbps}$ \\
Initial Backoff Window Size, WiFi - $C^{(W)}$ & 16 \\
Number of Sensing Slots, WiFi - $A^{(W)}$ & 2 \\
Maximum Backoff Stage, WiFi - $P^{(W)}$ & 6 \\
Retry Limit, WiFi - $Q^{(W)}$ & 1 \\
\hline Maximum Backoff Stage, 5G NR-U - $P^{(L)}$ & 6 \\
Retry Limit, 5G NR-U - $Q^{(L)}$ & 1 \\
MCOT duration, 5G NR-U & $8 m s$ \\
Slot duration, 5G NR-U - $D^{(L)}$ & $500 \mu s$ \\
\hline
\end{tabular}

uniformly distributed within $[0,1)$. We use SciPy's optimization tools [15] which provide an implementation of Sequential Least Squares Programming, where (18) is substituted by an equivalent linear least squares subproblem [14].

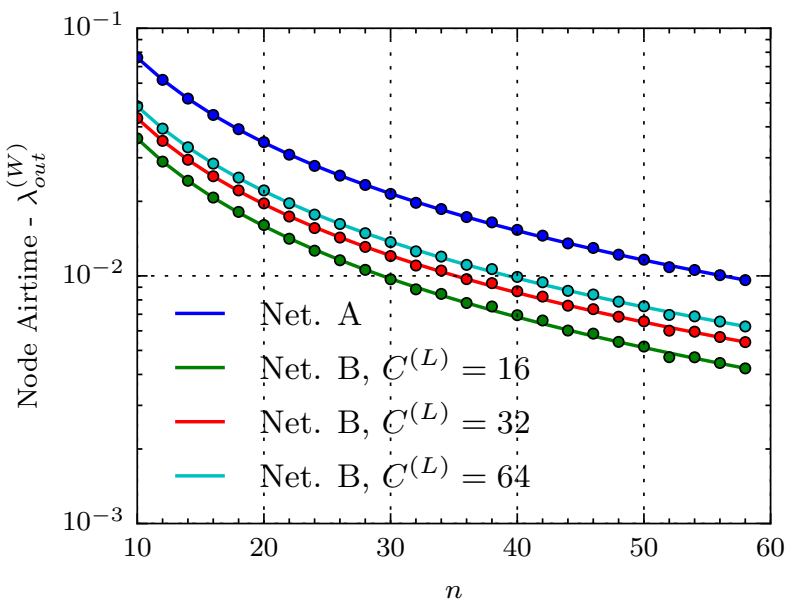

Fig. 4. Comparison of node airtime in networks A and B on varying $n$ with different values of $C^{(L)}$.

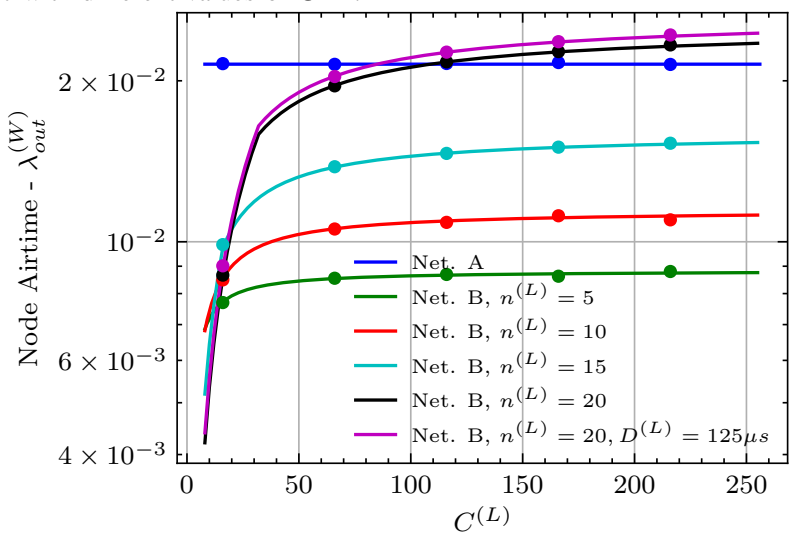

Fig. 6. Comparison of node airtime in networks A and B on varying $C^{(L)}$.

In each of the results, we compare the node airtime values of Networks A and B. When 3GPP fairness is not achievable, Algorithm 1 will generate the best possible parameters along with the difference in throughput as compared to the ideal case. While it is possible to use $D^{(L)}$ as an optimization parameter, our preliminary results suggested that the impact was minimal even when reduced to the lowest possible value $(62.5 \mu \mathrm{s})$. As it is a major point of difference between 5G NR-U and LTE-LAA, we have changed the parameter at some places to study its impact. Figs. 3-6 validate the feasibility of 3GPP fairness by tuning one parameter. In Fig. 3, we vary the total number of nodes in the network. For network $\mathrm{B}$ we consider $n^{(W)}=n^{(L)}$. We consider three different values of $A^{(L)}$. Increasing the number of sensing slots should intuitively imply limited opportunities for NR-U nodes to transmit and therefore increase $\lambda_{\text {out }}^{(W)} \cdot \lambda_{\text {out }}^{(\Psi)}$, in fact, is exponentially related to $A^{(\Psi)}$ [10]. We observe that 3GPP fairness cannot be achieved even when $A^{(L)}$ is as large as 8 . The results deviate when $A^{(L)}$ is large because of the non-homogeneity created due to the difference between the number of sensing slots. We gain a similar inference from Figs. 4 and 5. Fig. 4 depicts the variation of $\lambda_{\text {out }}^{(W)}$ with varying $C^{(L)}$. We observe that the difference in node airtime values is higher than Fig. 3, even at a fairly large value of $C^{(L)}=64$. In Fig. 5 we consider $A^{(L)}$ 


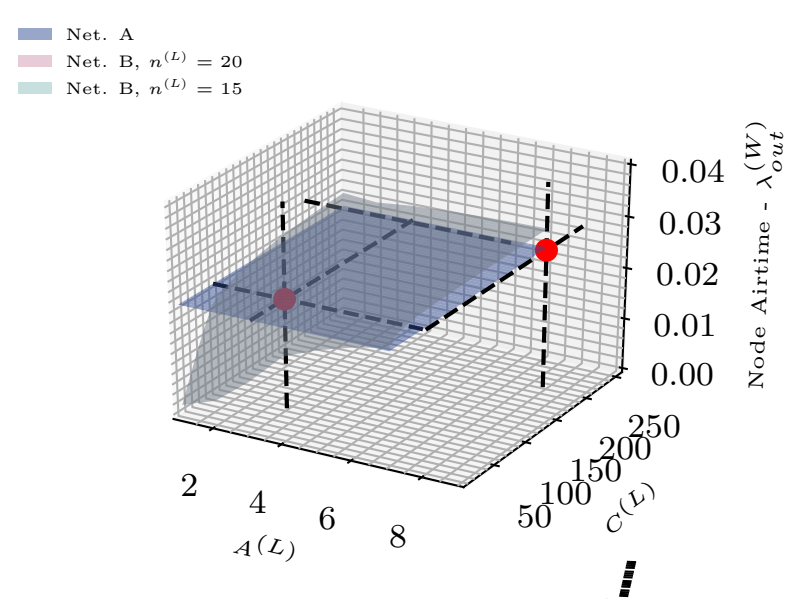

Fig. 7. Comparison of node airtime in networks $\mathrm{A}$ and $\mathrm{B}$ on varying $C^{(L)}$ and $A^{(L)}$.

as our independent variable such that $n^{(W)}+n^{(L)}=30$. The results are significantly far from 3GPP fairness even when the number of 5G NR-U nodes are increased to as many as $50 \%$ of total. Reducing the NR-U slot duration to $D^{(L)}=62.5 \mu \mathrm{s}$ which is the lowest possible causes some improvement in the throughput.

Fig. 6 deals with the variation against $C^{(L)}$ by changing $n^{(L)}$ such that $n^{(W)}+n^{(L)}=30$ so, where the graphs intersect at $n^{(L)}=20$. The point of intersection, which is at around $C^{(L)} \approx 109$ is accurately determined by Algorithm 1 . As a high value of backoff parameter will reduce the chances of an 5G NR-U node transmitting, its throughput is compromised. The high value can be traded off by reducing the slot duration. Considering $D^{(L)}=125 \mu s, C^{(L)}$ reduces to 85 . Further results consider tuning two parameters at a time. Algorithm 1, as discussed, supports tuning multiple parameters, which would be the case in a practical scenario. We vary $C^{(L)}$ and $A^{(L)}$ in Fig. 7. As Fig. 5 showed optimistic results for $n^{(L)}=20$, it is reasonable to expect similar outcomes. 3GPP fairness is achieved at $C^{(L)} \approx 58$ and $A^{(L)} \approx 3$, which are more reasonable parameters than what we achieved in Fig. 5. In Fig. 8, like Fig. 3 and 4, we consider $n^{(L)}$ and $n^{(W)}$. The surfaces do not intersect, but the distance is reasonably small at the values corresponding to $n^{(W)}=30$ and $n^{(L)}=1$. The difference is around 0.006 when $C^{(L)}=16, A^{(L)}=2$ and around 0.005 when $C^{(L)}=32, A^{(L)}=4$. The results indicate that 3GPP fairness is achievable if number of WiFi nodes are sufficiently higher than number of $5 \mathrm{G}$ NR-U nodes.

\section{CONClusion}

In this correspondence, we have formulated the problem of achieving 3GPP fairness as an optimization problem. We proposed how to use Sequential Quadratic Programming to tune 5G NR-U parameters to achieve fairness. The results show that the proposed method can be effectively used to predict the most suitable 5G NR-U parameters which are closest to satisfying the $3 \mathrm{GPP}$ criterion. It is also revealed that 3GPP fairness need not be achievable in general, and often when it is, either the 5G NR-U parameters are significantly larger than typical WiFi parameters, which would imply a reduced throughput, or the number of $5 \mathrm{G}$ NR-U nodes are

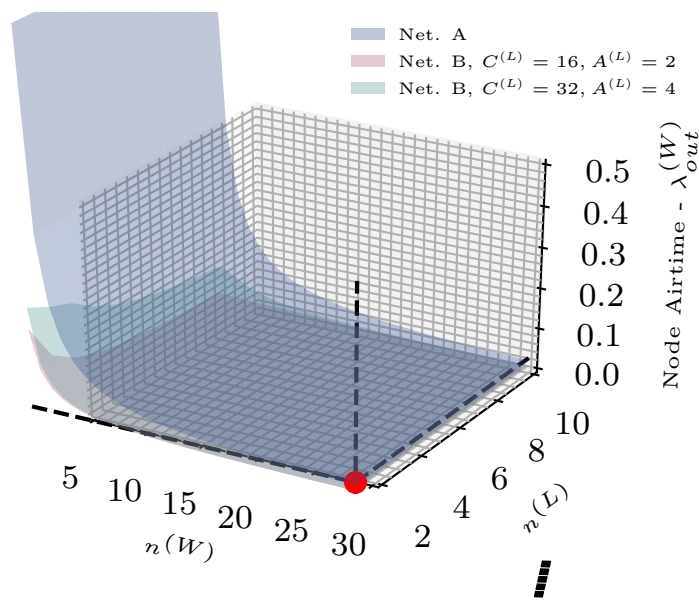

Fig. 8. Comparison of node airtime in networks A and B on varying $n^{(W)}$ and $n^{(L)}$.

significantly lower than $\mathrm{WiFi}$, or both. The proposed framework has proven useful in determining a suitable configuration for a fair coexistence of $5 \mathrm{G}$ NR-U and WiFi networks.

\section{REFERENCES}

[1] 3GPP, "Study on NR-based access to unlicensed spectrum; study on NR-based access to unlicensed spectrum (Release 16)," 3rd Generation Partnership Project (3GPP), Tech. Rep. 38.889, Dec. 2018, V16.0.0.

[2] _ - "Technical specification group radio access network; study on licensed-assisted access to unlicensed spectrum (Release 13)," 3rd Generation Partnership Project (3GPP), Tech. Rep. 36.889, Jun. 2015, V13.0.0.

[3] Y. Gao and S. Roy, "Achieving proportional fairness for LTE-LAA and Wi-Fi coexistence in unlicensed spectrum," IEEE Trans. Wireless Commun., vol. 19, no. 5, pp. 3390-3404, 2020.

[4] M. Mehrnoush, S. Roy, V. Sathya, and M. Ghosh, "On the fairness of Wi-Fi and LTE-LAA coexistence," IEEE Trans. Cogn. Commun. Netw., vol. 4, no. 4, pp. 735-748, 2018.

[5] S. Fang, Y. Gao, C. Zhang, and X. Hei, "Achieving 3GPP fairness for LTE-U and WiFi coexisting networks in unlicensed spectrum," in Proc. IEEE Int. Conf. Consum. Electron., 2019, pp. 1-2.

[6] V. Valls, A. Garcia-Saavedra, X. Costa, and D. J. Leith, "Maximizing LTE capacity in unlicensed bands LTE-U/LAA while fairly coexisting with 802.11 WLANs," IEEE Commun. Lett., vol. 20, no. 6, pp. 1219 $1222,2016$.

[7] J. Tan, S. Xiao, S. Han, Y. Liang, and V. C. M. Leung, "QoS-aware user association and resource allocation in LAA-LTE/WiFi coexistence systems," IEEE Trans. Wireless Commun., vol. 18, no. 4, pp. 2415-2430, 2019.

[8] M. Cierny, T. Nihtila, T. Huovinen, M. Kuusela, F. Chernogorov, K. Hooli, and A. Toskala, "Fairness vs. performance in rel-13 LTE licensed assisted access," IEEE Commun. Mag., vol. 55, no. 12, pp. 133-139, 2017

[9] J. Nocedal and S. J. Wright, Numerical Optimization. Springer, 2006.

[10] Y. Gao, X. Sun, and L. Dai, "IEEE 802.11e EDCA networks: Modeling, differentiation and optimization," IEEE Trans. Wireless Commun., vol. 13, no. 7, pp. 3863-3879, 2014.

[11] H. Bao, Y. Huo, X. Dong, and C. Huang, "Joint time and power allocation for 5G NR unlicensed systems," IEEE Trans. Wireless Commun., pp. 1-1, 2021.

[12] R. P. Brent, Algorithms for minimization without derivatives. Courier Corporation, 2013.

[13] P. Gawlowicz, A. Zubow, and A. Wolisz, "Enabling cross-technology communication between LTE unlicensed and WiFi," in Proc. IEEE INFOCOM, 2018, pp. 144-152.

[14] D. Kraft et al., "A software package for sequential quadratic programming," 1988.

[15] E. Jones, T. Oliphant, P. Peterson et al., "SciPy: Open source scientific tools for Python," 2001-. [Online]. Available: http://www.scipy.org/ 